\title{
Structural snapshots of Mycobacterium tuberculosis enolase during the reverse reaction reveal dual mode of 2PG binding and its implication in enzyme catalysis
}

\author{
Mohammed Ahmad ${ }^{1}$, Bhavya Jha ${ }^{1}$, Sucharita Bose ${ }^{2}$, Satish Tiwari ${ }^{1}$, Abhisek Dwivedy ${ }^{1}$, Deepshikha Kar ${ }^{1}$, Ravikant \\ Pal $^{1}$, Richard Mariadasse ${ }^{3}$, Tanya Parish ${ }^{4,6}$, Jeyaraman Jeyakanthan ${ }^{3}$, Kutti R. Vinothkumar ${ }^{5 *}$ and Bichitra Kumar \\ Biswal $^{1 *}$
}

\author{
${ }^{1}$ Structural and Functional Biology Laboratory, National Institute of Immunology, New Delhi-110067, India \\ ${ }^{2}$ Institute for Stem Cell Science and Regenerative Medicine, Bangalore 560065, India \\ ${ }^{3}$ Department of Bioinformatics, Alagappa University, Karaikudi, Tamil Nadu 630003, India \\ ${ }^{4}$ Infectious Disease Research Institute, 1616 Eastlake Avenue E, Suite 400, Seattle, WA98102, USA \\ ${ }^{5}$ National Centre for Biological Sciences, TIFR Bangalore 560065, India \\ ${ }^{6}$ Seattle Children's Research Institute, Seattle, WA 98109, USA
}

ahmad@nii.ac.in

Enolase is a ubiquitous enzyme found in the glycolytic pathway of organisms of all three domains of life and is involved in the catalysis of the reversible conversion of 2-Phosphoglycerate (2-PG) to Phosphoenolpyruvate (PEP). It plays an important role in Mycobacterium tuberculosis (Mtb) virulence by acting as cell surface receptor of human plasminogen. Enolase in Mtb stands out from most of its homologs by being catalytically active only in an octameric state. While the forward reaction is well understood, not much is known about how the catalytic conversion of PEP to 2PG takes place. Here we present structural snapshots of Mtb enolase (MtENO) at various stages as it progresses through the reverse reaction. We found a plausible catalytic pathway involving a novel transient product bound state called 'alternate conformation', in addition to the canonical one. We observed two major deviations from the forward reaction: presence of $\mathrm{Mg}_{\mathrm{B}}$ is non-obligatory for the reaction and flipping of $2 \mathrm{PG}$ to an alternate conformation makes it energetically feasible to exit the site. Molecular dynamics and free energy calculation further indicate that alternate conformation may act as exit conformation and facilitate the opening of active site loops due to distortion in metal ion coordination and H-bond interactions. Additionally, P-P docking and simulation study of enolase and plasminogen helps us to understand the molecular interaction of the complex.

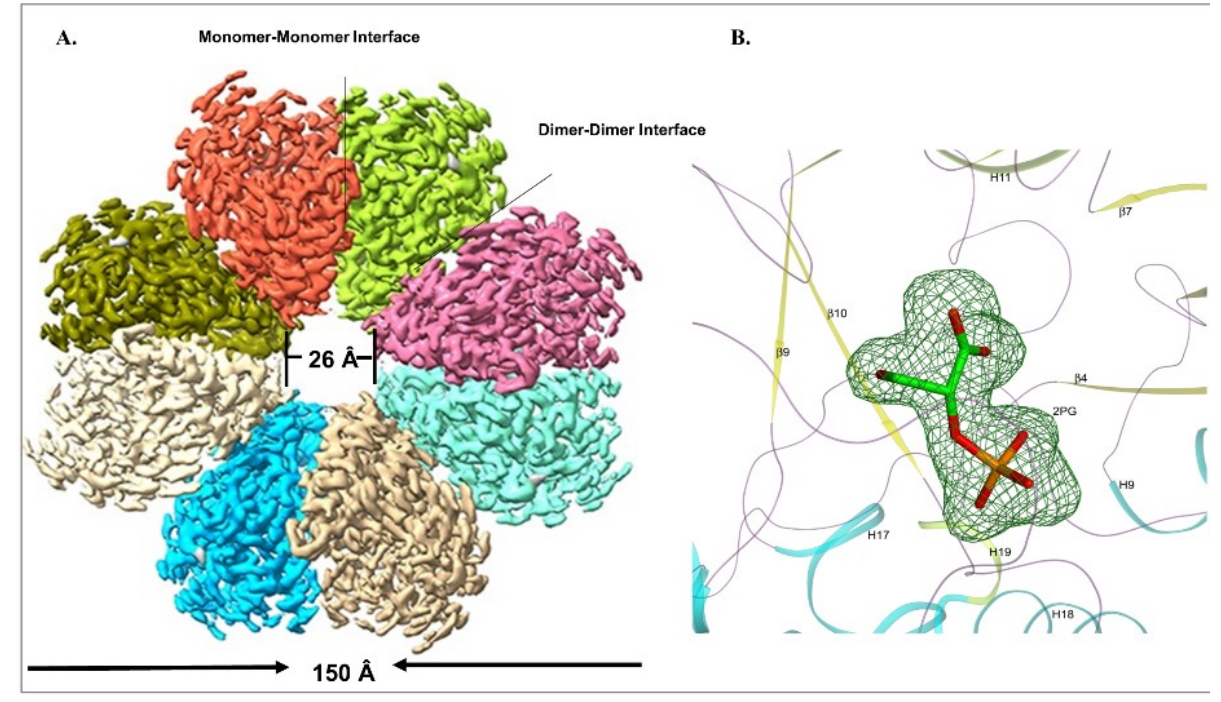

Figure. 1. (A) CryoEM structure of apo enolase (B) FoFc map at $3 \sigma$ of novel alternate conformation of 2PG.

Keywords: Mycobacterium tuberculosis, Enolase, CryoEM, X-ray crystallography, In-silico study 